\title{
El ejército azul: incidencia de la MINUSTAH en la estabilidad y seguridad del Estado haitiano*
}

\section{The blue army: MINUSTAH's incidence in stability and security of Haitian state}

\author{
Diego Fernando Prieto Olivares** \\ Fecha de recepción: 4/08/2017 \\ Fecha de aceptación: 27/09/2017
}

\section{Resumen}

Haití ha sido catalogado como un Estado fallido, debido a que en su historia reciente ha permanecido una constante intervención internacional, producto de la inestabilidad política y el control de las bandas criminales que hay dentro del territorio. La última misión desplegada en el territorio haitiano y que continúa vigente

* $\quad$ +Artículo de reflexión, que realiza una mirada sobre la incidencia de la misión de paz MINUSTAH en Haití durante un periodo de tiempo determinado a través de la teoría de la construcción de paz liberal.

** +Estudiante de Relaciones Internacionales de la Universidad de San Buenaventura, sede Bogotá. Se ha desempeñado como semillerista en el semillero Comisiones de la verdad, adscrito a la Facultad de Ciencias Jurídicas y Políticas de la misma Universidad y ha sido ha sido auxiliar de investigación del proyecto Get the truth out of truth commissions. Correo Electrónico: diegop_0@hotmail.com 
en la actualidad es la Misión de Estabilización de Naciones Unidas en Haití (MINUSTAH), cuyos objetivos primordiales están en mantener la paz y la seguridad en Haití, así como apoyar un proceso político adecuado y congruente para construir la institucionalidad haitiana. Por tanto, dado que la MINUSTAH ha tomado un papel de ejército azul, el presente artículo busca investigar las implicaciones que ha tenido dicha misión en el mantenimiento de la seguridad, así como en el apoyo al proceso político y la situación de derechos humanos, tomando como punto referente la teoría de la construcción de la paz liberal.

\section{Palabras clave}

Construcción de paz liberal, Estado fallido, Haití, MINUS$\mathrm{TAH}$, misiones de paz, seguridad humana.

\section{Abstract}

Haiti has been categorized as a failed stated, because of a constant international intervention in it's recent history, produced by political instability and control of criminal gangs. The last peace mission that had been deployed, and has validity nowadays, is the United Nations stabilization mission in Haiti (MINUSTAH), with objectives as maintain the peace and security in Haiti, support the political process for building and Haitian institutionally. In that way, and taking account MINUSTAH has taken a Blue army role, this article pretend study the implications MINUSTAH has been in the maintaining of security, the support of the political process and the human rights situation, taking as a theoretical reference the liberal peacebuilding. 


\section{Key words}

Haiti, Liberal peacebuilding, Failed state, human security, MINUSTAH, peace missions.

\section{Introducción}

La Organización de Naciones Unidas (ONU) ostenta como sus mejores estandartes, el mantenimiento de la paz y la seguridad internacionales, así como el respeto por la igualdad y los Derechos Humanos. No obstante, cuando se hace una revisión a fondo de los resultados que han arrojado las 68 misiones de paz que van hasta la actualidad, se evidencia una serie de inconsistencias dentro del mantenimiento de la paz en los lugares donde han sido desplegadas (Dural, 2013). Homicidios, intimidación y violencia sexual son algunos de los aspectos que se han presentado en las misiones de paz y que van en contravía de su fin último.

Igualmente, dichas misiones de paz pueden ser interpretadas como intervenciones en busca de fortalecer valores democráticos presuntamente aceptados globalmente. Tomando la interpretación de Valdés (2008), las misiones de paz pueden encontrar choques entre interventores e intervenidos; los primeros, considerarán que están actuando correctamente en beneficio de un territorio desequilibrado y con necesidad de control. Los segundos, entretanto, verán como invasores e ilegítimos a quienes intervengan en su territorio, sin importar si están siendo respaldados por la comunidad internacional. Así, en medio de tal choque de posiciones, pueden generarse situaciones que se apartan de la construcción de paz que busca una misión de esta categoría.

Por tales motivos, es importante estudiar las condiciones en las que se envía una misión de paz, y revisar la incidencia que esta ha tenido en el logro de su mandato. El caso de Haití es importante a 
tratar, debido a que es un Estado catalogado como fallido y que ha tenido constantes intervenciones por parte de la comunidad internacional, de acuerdo con Serrano (2007). Un Estado fallido, a su vez, es definido como uno que no es capaz de mantenerse como un miembro de la sociedad internacional y que puede poner en peligro a sus Estados vecinos a causa de la inestabilidad política, conflictos, revoluciones, migraciones constantes de refugiados, entre otros, (Zapata, 2014).

Haití entra así en la definición de Estado Fallido presentada por Zapata (2014), dada la constante inestabilidad política que ha vivido a lo largo de su historia y que será referenciada más adelante en el presente texto. No obstante, a pesar de su inestabilidad, solo en el periodo entre 2003 y 2004 ocurrió una crisis violenta de tal magnitud, que llegó a ser catalogada como conflicto armado y a consecuencia fue enviada por la ONU la Misión de Estabilización de Naciones Unidas en Haití (MINUSTAH), para controlar cuestiones de seguridad y vigilar el correcto desenvolvimiento de un gobierno interino.

\section{Marco Teórico}

iAl catalogarse como el primer Estado fallido de América, Haití se ha convertido en el punto de mira de académicos que han buscado analizar la situación por la que atraviesa dicho país. Valdés (2008) se enfoca en observar tanto la percepción que tuvo la sociedad haitiana a la misión de paz, como las labores de la MINUSTAH en el ámbito político y militar. Indica que muchas facciones de la sociedad haitiana consideran a la misión de paz como invasiva, como una forma de forzar la paz. Entretanto, otros consideran que, aunque la misión no debería ejercer acciones políticas, sí es necesaria para establecer seguridad dentro del país. 
Serrano (2006) se refiere primero a la historia política de Haití desde su independencia hasta el 2007, haciendo un recuento del aislamiento internacional que padeció en un principio, para luego observar la serie de dictaduras e intervención internacional, que se fueron dando a lo largo del siglo XX. A diferencia de Valdés, la autora no solo se enfoca en los esfuerzos de la MINUSTAH sino en todos los procesos de intervención que se dieron en Haití. En total, desde 1993 hasta 2004, se dieron cinco misiones de intervención en Haití, culminando con la MINUSTAH, cuyos objetivos rondan en tres aspectos principales: generar estabilidad y seguridad, apoyar el gobierno de transición y garantizar el respeto por los derechos humanos.

González y Manero (2011), por otra parte, no solo generan un análisis de las intervenciones de paz en Haití como lo hace Serrano, ni se enfocan exclusivamente en la MINUSTAH como lo hace Valdés, sino que generan un análisis integral del contexto histórico de Haití, junto con todos los factores socioeconómicos y políticos que condujeron al país a la situación en la que se encuentra en el 2011. También, toman en cuenta las intervenciones de paz e incluso un análisis sobre la situación de Haití luego del terremoto del 2010. Las conclusiones extraídas por los autores resultan desoladoras para el futuro del país, ya que indican que es un país sin estructuras del poder, con una historia que lo ha puesto en una situación caótica y con elementos medioambientales que lo asolan cada cierto tiempo.

Dejando el estado del arte sobre la temática elegida para el presente trabajo y entrando en materia teórica, se tiene que el prisma más adecuado para observar el estudio de caso haitiano es la construcción de paz liberal (o liberal peacebuilding). De acuerdo con Paris (2010), la construcción de paz liberal es una herramienta que ayuda a los Estados a emerger luego de contextos de guerra civil. Una herramienta, no obstante, que ha sido objeto de constantes críticas, 
debido especialmente, al decepcionante ratio de éxito que ha manejado. Las críticas también se enfocan en afirmar que dicha construcción de paz liberal busca expandir valores democráticos en una suerte de neocolonialismo e imperialismo.

No obstante, Paris (2010) objeta las críticas afirmando que es necesario clarificar el concepto de paz liberal y liberalismo, que es de por sí un concepto problemático y que, asociarlo a la construcción de paz puede tener efectos adversos. Paris indica que es necesario reconocer que la aplicación de la construcción de paz y las misiones han tenido mayores efectos positivos que negativos, y que, dejar de utilizarlos implicaría dejar en desamparo a más de 10 millones de personas. También, haciendo contraposición a las críticas de los académicos, indica que es falso realizar una analogía entre la construcción de paz liberal y la guerra estadounidense contra el terror, así como los procesos de colonización que se tuvieron en el pasado.

Roberts (2012), a diferencia de Paris, no pretende defender a la construcción de paz liberal, sino que le da duras críticas. De acuerdo con el autor, cerca de la mitad de los procesos de construcción de paz liberal demuestran pocas prácticas democráticas más allá de la ejecución de unas elecciones y la creación de comisiones de la verdad y otros instrumentos de justicia transicional. Igualmente, muchos de los territorios que son intervenidos en busca de la construcción de paz retornan a situaciones de violencia tan pronto como los interventores se van, dado que no dejan bases suficientes para generar cambios estructurales que pongan fin a la situación conflictiva.

Como segunda parte del presente marco teórico, se dan las aseveraciones teóricas respecto a la seguridad humana, que de acuerdo a Fuentes (2012), aparece oficialmente en la escena internacional, gracias al informe del Programa de las Naciones Unidas para el Desarrollo (PNUD). Desde entonces, se han dado numerosos debates sobre las aseveraciones y alcances del concepto, entre las cuales se encuentra el 
concepto dado por el Consejo de Seguridad Humana, en el que indica que esta es protección de la esencia de la vida humana, el realce de sus libertades fundamentales y la plena realización del ser humano.

Dicho concepto implica una amplia variedad de interpretaciones; no obstante, también Fuentes (2012) indica la existencia de cinco principios que permiten entender de mejor manera el concepto. Primero, el concepto de la seguridad humana se centra en las personas, por tanto, busca entender y minimizar los riesgos a la supervivencia, los medios de vida y la dignidad de estas. Segundo, es multisectorial, lo que quiere decir que implica el entendimiento de diversas fuentes de riesgos para el ser humano, entre los que se cuentan la economía, la salud, la alimentación, el medio ambiente y la seguridad personal, comunitaria y política, así como la interconexión entre estas amenazas.

Tercero, es integral, por lo que requiere que la solución a las problemáticas sea una que abarque cada uno de los aspectos multisectoriales mencionados en el anterior apartado. Cuarto, es contextualizada, lo que indica que los riesgos e inseguridades pueden variar (y por ello aumentar o disminuir) de acuerdo a determinado contexto. Quinto, es preventivo, de forma que se centra en llegar a las causas de las inseguridades y riesgos para introducir estrategias de empoderamiento que mitiguen los posibles riesgos (Fuentes, 2012).

Ambos aspectos teóricos (liberal peacebuilding y seguridad humana) se encuentran y entrechocan en el caso haitiano, dado que las consecuentes intervenciones que se han dado en el país no han conseguido una mejora significativa de la violencia ni de los problemas estructurales a los que se enfrenta Haití, y la seguridad de sus habitantes ha tomado numerosos riesgos (tanto de índole política como en temas de seguridad personal, económica y alimentaria), dada la situación de inestabilidad por la que atraviesa el país, especialmente, durante la primera década del siglo XX. Por tanto, es preciso 
observar las consecuencias que ha tenido el último mecanismo de intervención en el país, de forma que se mire si ha tenido o no resultados positivos, al menos en lo referente al tema de la seguridad de sus habitantes. De allí, surge la siguiente pregunta de investigación: ¿cuáles fueron los principales efectos del establecimiento de la MINUSTAH en Haití desde 2004 hasta 2010 en materia de seguridad humana?

\section{Haití: un Estado y una paz aún en construcción}

Durante toda la historia de Haití como república independiente, ha pasado por una situación política enmarcada en la inestabilidad. No obstante, en su historia reciente, ha sido mucho más notoria la debilidad del Estado para hacer frente a situaciones de seguridad pública, crecimiento económico y otros aspectos que han afectado su desarrollo como nación. En la segunda mitad del siglo XX, la mayoría del tiempo estuvo dominado por una de las dictaduras más sangrientas que haya podido tener en toda su historia, con JeanClaude Duvalier (1957-1986) y es solo hasta finales de la década de los ochenta, que empieza a buscar la forma de desarrollar procesos democráticos (Wargny, 2005).

Para diciembre de 1990, se da uno de los primeros intentos de tornar a la democracia con las elecciones, que fueron ganadas por el presidente Jean Bertrand Aristide, con un 67\% de los votos. Esto, no obstante, no fue del agrado de la cúpula militar haitiana que, de acuerdo con Wargny (2005), orquestó en 1991 un golpe de estado exitoso, apoyado en buena parte por Francia y Canadá. Dicho golpe de Estado desembocó en una junta de militar que impidió las reformas que en un principio había propuesto Aristide, durante su campaña; sin embargo, es repuesto en el poder gracias a operaciones apoyadas por la marina estadounidense en 1994, donde empieza su segundo mandato presidencial, que tendrá lugar hasta 1996. Es quizá este periodo histó- 
rico el que posee una mayor relevancia en la situación actual haitiana, dado que es durante esta presidencia que Aristide:

Delegó las tareas de seguridad y represión a bandas armadas paradelincuenciales, permitió el auge del narcotráfico y la corrupción mientras se derrumbaba la economía, y agudizó la polarización social con un uso habitual de la violencia política y del fraude electoral (Wargny, 2005, p. 2).

Posteriormente a Aristide, las nuevas elecciones dejan en el poder a René Preval para tener su primer mandato presidencial. Dicho mandato se da bajo las condiciones puestas por Aristide y consigue terminarlo (siendo el primer presidente electo en terminar su mandato presidencial), para dar lugar a unas nuevas elecciones que culminarían el siglo XX. La Misión Internacional de Apoyo Civil en Haití (MICAH), como fuerza multinacional de paz que había sido desplegada en el país para el apoyo de las instituciones haitianas, es retirada con la esperanza de que las elecciones fueran suficientes para estabilizar el país (González y Manero, 2011). No obstante, la situación política posterior a estas se convirtió en una mucho más delicada y compleja.

Con la victoria de Fanmi Lavalás en el año 2000 (bajo la tutoría política de Aristide) y con las graves acusaciones de fraude provenientes de la oposición haitiana, la tensión en el país se hacía cada vez mayor. La tensión política, junto con las bandas delincuenciales que habían sido armadas por los partidarios de Lavalás, generaron una creciente situación de violencia. Para el 2001, por diversas causas, Jean Bertrand Aristide retorna a la presidencia de Haití, lo que genera mayores situaciones de tensión; pero es solamente hasta el año 2004, con el bicentenario de la independencia de Haití, que empezó la situación de extrema violencia en el país, que llegó casi a tratarse de un conflicto armado (González y Manero, 2011).

Para el adecuado entendimiento de un conflicto armado (o de una situación de alta violencia interna como lo fue el caso de Haití), 
existen numerosos instrumentos y entre ellos se encuentran los distintos modelos de mapeos propuestos por Mason y Rychard (2005). Entre dichos mapeos, el instrumento que sirve para entender las causas estructurales de un conflicto y la forma en que dichas causas se vuelven manifiestas es el Árbol de conflictos, que se presenta más adelante en el gráfico 1. Wargny (2005), indica que aunque Haití no se ha calificado como un conflicto armado, cuenta con varias características propias de uno, como lo son la existencia de índices de letalidad vinculados al conflicto armado (o en este caso a la situación de violencia extrema), presencia de grupos armados, una operación de mantenimiento de la paz, circulación de armas ilegales y ayuda para la rehabilitación posbélica.

Gráfico 1: Árbol de conflictos de Haití

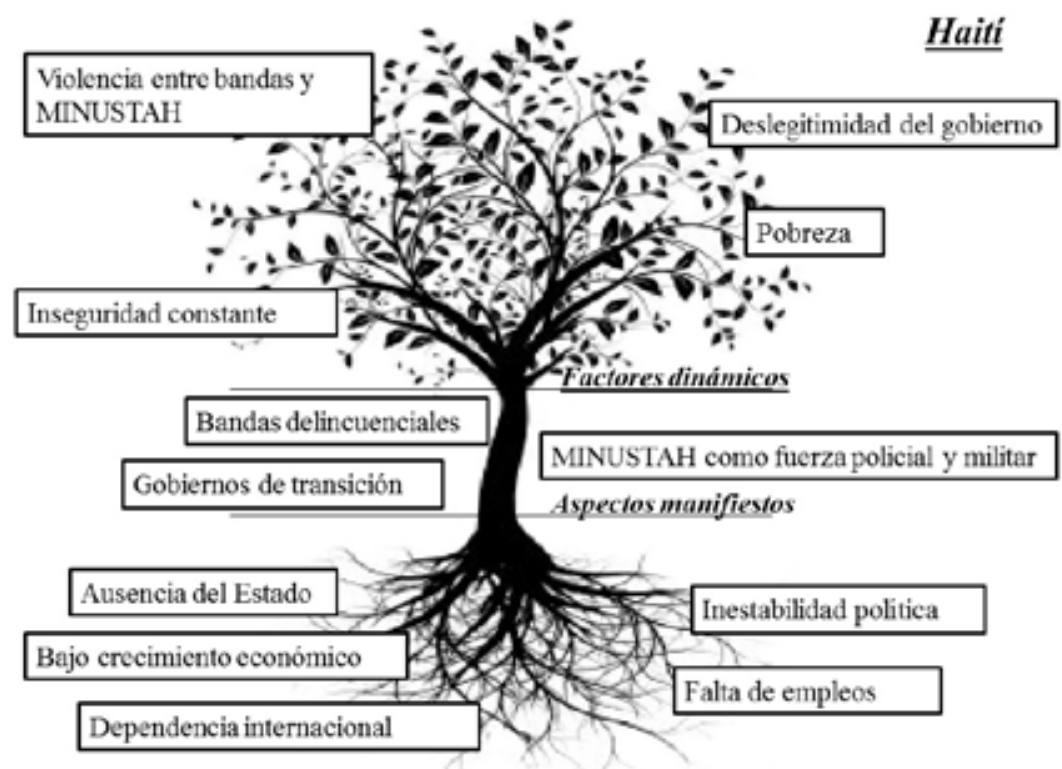

Factores estructurales

Fuente: Elaboración propia basada en Wargny (2005) y

González y Manero (2011). 
Continuando entonces con las causas del conflicto de facto haitiano mostradas en el gráfico 1 , se tiene que, parte de las causas estructurales están en la constante desestabilidad política que ha vivido el país, que ha derivado en una invariable ausencia del Estado en buena parte del territorio nacional. A dichas causas, a su vez, se adjuntan una firme crisis económica, falta de empleos y dependencia a las ayudas de la comunidad internacional. De acuerdo a González y Manero (2005), durante un tiempo de la historia de Haití, las inversiones extranjeras de las que dependía optaron por retirarse del país, dejándolo en un estado de desolación y desesperanza para el futuro.

De dichas causas estructurales se desprenden los aspectos manifiestos observados en el árbol. De acuerdo con Wargny (2005), un buen número de bandas delincuenciales se tomó los territorios donde el Estado no tenía presencia, dada su debilidad institucional. Parte de la articulación de dichas bandas criminales está en la falta de empleos vista en las raíces del árbol, como una forma de subsistir en un ambiente hostil, donde se busca la mejor manera de sobrevivir. A raíz de la situación presentada en Haití, se despliega la MINUSTAH como la última intervención que ha tenido lugar en el país, cuyo mandato afirma que está para establecer la seguridad y el apoyo al gobierno de transición, pero que acaba convirtiéndose en un ejército azul, en lucha contra la desarticulación de las bandas criminales.

Conviene tener en cuenta que, de acuerdo a lo mencionado por Couceiro (2005), no se puede hablar de un ejército dado que durante el segundo mandato de Jean-Bertrand Aristide (1994-1996) se abolió el ejército, pero no se desarmó a sus miembros. Por tal motivo, se habla también de grupos paramilitares que entran como actor en el «conflicto» haitiano, mientras que la MINUSTAH funge como ejército en apoyo del gobierno de Haití, desde al año 2004, fecha del establecimiento de su mandato. Puede decirse que la intervención persistente de la comunidad internacional en Haití pone 
de manifiesto los esfuerzos de esta en la construcción de paz dentro del país.

Por otra parte, es pertinente revisar verdaderamente qué tanto interés existe por parte de la comunidad internacional en una verdadera construcción de paz en Haití. Antes de la creación de la MINUSTAH, la inestabilidad política que se mencionó anteriormente se hizo presente, en parte debido a que Jean-Bertrand Aristide, quien, anteriormente, había sido sacado de su puesto por un golpe de Estado, fue restablecido en este por miembros de la armada estadounidense (Wargny, 2005). Posteriormente, en 2004, fue también objeto de un golpe de Estado y fue sacado de manera exitosa en una operación militar comandada por Estados Unidos, Francia, Canadá y Chile. Conviene tener en cuenta, además, que Aristide tenía antecedentes de ser un gobierno corrupto y en contacto con el tráfico de drogas y armas.

La MINUSTAH opera desde junio del año 2004, y su mandato se enfoca en tres aspectos principales, a saber: la estabilización y seguridad de Haití, el apoyo al gobierno de transición y la protección de los derechos humanos (Couceiro, 2005). Igualmente, luego del año 2005, la MINUSTAH contaba con cerca de 8.700 efectivos, entre los cuales, el $72 \%$ del personal era militar; entretanto, un $16 \%$ eran policías y un $10 \%$ era población civil. La composición de la misión, de acuerdo a Couceiro (2005), estaba en un contingente de 20 países, de los cuales solo cinco eran países del «Norte». El mando de la MINUSTAH era brasileño y casi la mitad de las nacionalidades que conformaban el contingente eran latinoamericanas.

Esto afianzó parte de la confianza del pueblo haitiano hacia la MINUSTAH. Las anteriores intervenciones por parte de la comunidad internacional (y especialmente por parte de Estados Unidos), socavaron dicha confianza en las intervenciones de países desarrollados. A su vez, Estados Unidos tampoco tenía demasiado interés en 
generar una nueva intervención en la isla, dados los malos resultados que había tenido en su intervención en Somalia, en el marco de la misión de paz, donde se perdieron las vidas de un considerable contingente de soldados estadounidenses (Torchiaro, 2007).

Fue esa la principal razón para que el proceso de la MINUSTAH no estuviera abanderado por Estados Unidos (ni que tuviera realmente un componente militar, pese a que sí hubo presencia estadounidense dentro de la misión). Por otra parte, de acuerdo a Torchiaro (2007), Francia y Canadá, que habían mantenido lazos importantes con Haití, dados sus parecidos en materia cultural y lingüística, tampoco tuvieron una participación muy protagónica dentro de la MINUSTAH, dado que la comunidad internacional mantenía en ellos una suerte de condena tácita, por haber colaborado con la salida del poder de Jean Bertrand Aristide (un presidente electo democráticamente, aún a pesar de ser acusado de corrupto y en contacto con el tráfico de dogas) durante la crisis y el golpe de Estado del 2004.

De acuerdo con Couceiro (2005), la MINUSTAH fue criticada desde su primer año. Por una parte, los haitianos la consideraban como una injerencia del exterior en su propio país, y no tenían conocimiento alguno de su mandato, de forma que desconocía las razones por las cuales se encontraba en su territorio, así como la misión que debía cumplir. A lo anterior, se suma que muchos de los miembros contingentes de la MINUSTAH no hablaban ninguna de las lenguas del territorio: el francés ni el creole. Durante el primer año, la MINUSTAH fue criticada por haber tenido una conducta más bien pasiva, pese a que se indicaba que la fuerza no serviría para mejorar la situación a largo plazo, sí servía al menos para estabilizar el país. De igual forma, algunos llegaron a calificar a la MINUSTAH como un instrumento cuya única actividad era entrar a las ciudades a disparar, bajo excusa de luchar contra las bandas delincuenciales; no 
obstante, dicha actividad solo dejaba saldos de muertos de población civil, más que una verdadera lucha contra las bandas.

Luego del primer año de acciones de la MINUSTAH, se dan enfrentamientos entre los cascos azules y antiguos miembros del disuelto ejército haitiano, ahora miembros de los mencionados grupos delincuenciales. La lucha contra dichas bandas y el despliegue de fuerzas se hace mucho más activa, luego de la muerte de dos soldados de la misión en Haití, en marzo de 2005, producto del fuego cruzado entre estos y las bandas criminales. Después de esto, y a pesar de las críticas que ha habido frente a la muerte de civiles en el fuego cruzado, la MINUSTAH ha colaborado en la situación en Haití y ha conseguido controlar de cierta forma la violencia por parte de las bandas criminales (González y Manero, 2011).

No obstante, dicho control ha tenido un alto precio en cuestión de vidas civiles haitianas. Tal y como se observa en el gráfico 2 , se mantiene una línea progresiva de homicidios intencionales desde el año 2007 (que es el año desde el que se ofrecen las cifras en el banco mundial respecto a Haití), hasta el año 2012; de tal forma que la MINUSTAH, si bien ha cumplido parte de su mandato, continúa manejando una cierta debilidad frente a las bandas criminales y los aspectos de seguridad de la población.

Es muy probable, a su vez, que el alza exponencial de los homicidios intencionales desde el 2010 esté estrechamente ligado con el terremoto que arrasó con Haití en dicho año. Este tema, que no es el eje central del presente trabajo de investigación, pero que debe tenerse en cuenta en el análisis de la gráfica presentada, supone un cambio en toda la estructura de la MINUSTAH y en su accionar frente a la sociedad haitiana. Por tal motivo, se da como punto de quiebre a la pregunta de investigación el año 2010, puesto que el terremoto de Haití supone todo un nuevo tema de estudio y cambio de perspectiva del accionar de la MINUSTAH. 
Gráfico 2. Número de muertes intencionales anuales en Haití.

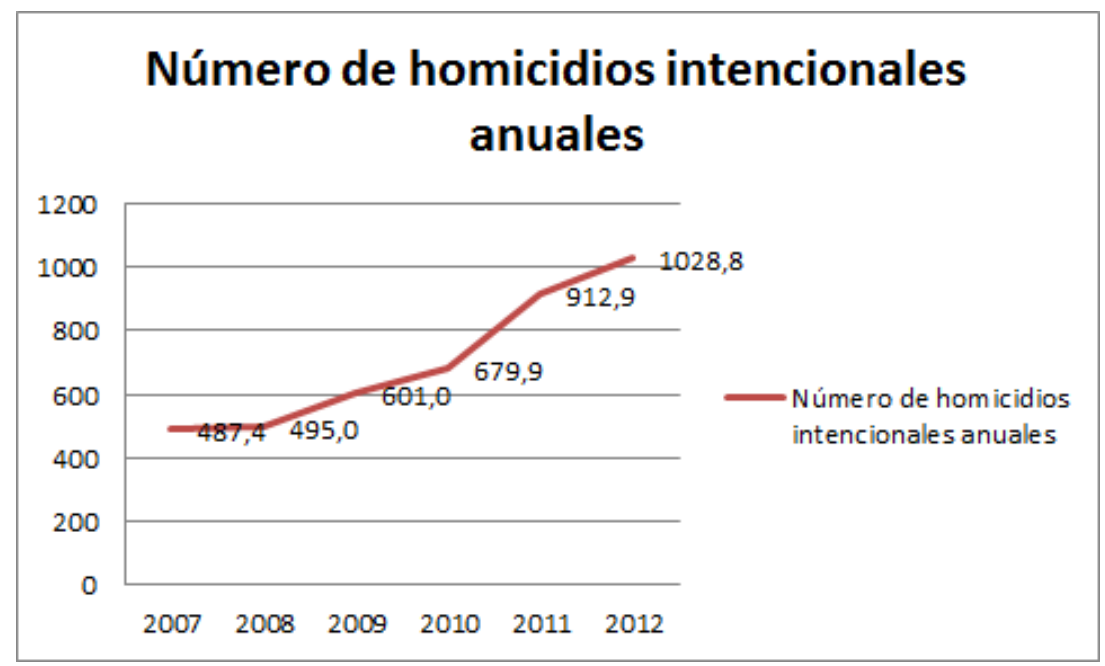

Fuente: Elaboración propia a partir de datos sobre homicidios por cada 100.000 habitantes y datos sobre población anual del Banco Mundial, 2016.

Cabe destacar, igualmente, que buena parte de los homicidios mostrados en la gráfica, no son solamente adjudicadas a las bandas delincuenciales que rondan por todo el territorio haitiano, sino también a los miembros de la MINUSTAH y de la Policía Nacional Haitiana $(\mathrm{PNH})$. Las mayores críticas que ha tenido la misión se centran en las constantes violaciones a los derechos humanos, que han tenido lugar por parte de activos de la misión y que han sido, en algunos casos, denunciados ante la comunidad internacional.

De acuerdo con Grandin y Bhatt (2011), cerca de 8000 personas (de las cuales la gran mayoría era partidaria de Jean Bertrand Aristide), fueron asesinadas o desaparecidas durante el tiempo en el que la MINUSTAH debía asegurar su protección. Igualmente, refieren el caso de un joven haitiano de 18 años que fue asaltado sexualmente por miembros uruguayos de la misión, que fueron inmediatamente 
repatriados para esperar acciones legales en prisión. No obstante, de acuerdo a los autores, este fue solo uno de los excepcionales casos en que los soldados de la MINUSTAH fueron juzgados y procesados, dado que la misión posee una larga lista de violaciones de derechos humanos que permanecen en la impunidad.

Para el año 2007, un contingente de al menos 100 soldados provenientes de Sri Lanka debió ser repatriado también debido a su vinculación con abusos y malas conductas sexuales. Dichos soldados habrían estado involucrados con explotación sexual que involucraba a jóvenes menores de edad, que fueron obligadas a intercambiar sexo por comida, dinero o incluso teléfonos celulares. Del caso no se tiene evidencia alguna de que los soldados repatriados hayan sido procesados, lo que socaba notablemente la legitimidad de la MINUSTAH y su contingente humano (Grandin y Bhatt, 2011).

Por otra parte, la seguridad humana en Haití (desde varios de sus múltiples componentes), no solo se ha visto puesto en riesgo debido a la inseguridad que representa la presencia de los soldados en el territorio, sino por la subsecuente situación de salud pública que ha tenido lugar a causa de la misma misión. El virus del Cólera, de acuerdo a Grandin y Bhatt (2011), fue introducido en Haití por soldados nepalíes que provenían de la MINUSTAH y que expandieron el virus por medio de un mal tratamiento de residuos y la contaminación de las aguas de los ríos. De acuerdo a los autores, aun cuando en caso de que no le sean imputados cargos por responsabilidad a la misión (y por ende a la ONU), si se pueden imputar cargos a la misma por negligencia y omisión de la crítica situación de salud pública.

Es así como se observa un fracaso evidente en la consecución de su tercer objetivo primordial, referente a la protección de los derechos humanos. El referente a la estabilización y seguridad de Haití se ve a su vez cumplido solo en una pequeña parte, donde a través de ciertos programas se busca la estabilidad dentro del país. Un ejem- 
plo de esto son los esfuerzos en materia de Desarme, Desmovilización y Reintegración (DDR). Dentro de las estrategias que tiene la MINUSTAH para el programa de DDR está la oferta de ayudas y beneficios a sectores que se desarmaran y reinsertaran a la sociedad. Igualmente, han surgido otras alternativas, tales como talleres de capacitación a los partidos políticos en temáticas de derechos humanos, dado el creciente número de violaciones y explotación infantil, así como una línea de denuncias para las víctimas (González y Manero, 2011).

Aun cuando se han generado diversas alternativas para desmovilización, han sido limitados los avances para la erradicación de las causas estructurales del conflicto haitiano de facto. Lo anterior, puede entenderse bajo la lógica (muy común en las misiones de paz de la $\mathrm{ONU}$ ) de que es necesario primero establecer un régimen democrático y asegurar unas elecciones legítimas para luego enfocarse en la reconstrucción a partir de un gobierno estable. Por tanto, de acuerdo con lo mencionado por Feldmann y Montes (2008), la MINUSTAH ha conseguido al menos uno de sus objetivos primordiales, saber, la realización de elecciones con un alto porcentaje de legitimidad en el territorio.

La elección que aseguró la llegada de René Preval al poder haitiano en su segundo mandato, supuso un importante avance en materia política y un logro significativo para la MINUSTAH en el cumplimiento de su objetivo referente al apoyo de la democracia en Haití. En un ambiente más bien hostil, en el que había constantes luchas y problemas de inseguridad a lo largo de todo el territorio, así como un ambiente de fragmentación política, que las personas salieran a ejercer sus labores democráticas fue visto como una victoria en sí misma. Más aún, lo fue observar que la mayoría de la población aprobaba al candidato que fue elegido y lo veían como algo legítimo. Esto supuso un enorme paso para la estabilización gubernamental 
de Haití, aun a pesar de los problemas que seguiría enfrentando en materia de causas estructurales.

Dicho proceso democrático, apoyado por una fuerza internacional como la MINUSTAH es, esencialmente, importante debido a que la sociedad desaprueba una reforma y un cambio estructural siempre que vea que esta irá en detrimento de su diario estilo de vida. En el caso contrario, la sociedad legitima un proceso si observa que este se convierte en una mejora de su situación y observa un verdadero cambio que le brinden oportunidades de crecimiento. Fue así, de acuerdo con Le Chevalier (2011), como la sociedad haitiana observó las elecciones tenidas lugar en el año 2006. A su vez, la MINUSTAH continuó ejerciendo sus labores de pacificación y de entrenamiento a la fuerza policial, de forma que se continuase con la consecución de los objetivos que tuvo al momento de su despliegue.

\section{Conclusiones}

Los efectos que tuvo el proceso de la MINUSTAH en materia de seguridad humana en Haití deben verse desde diversas aristas, precisamente, por la integralidad y multisectorialidad del concepto. Por una parte, la lucha que tuvo la MINUSTAH en conjunto con la PNH para el combate de las bandas delincuenciales tuvo, en partes iguales, consecuencias beneficiosas y perjudiciales para la seguridad humana de los haitianos. Los beneficios (que pueden no verse tan explícitos) pueden verse en que, gracias a la desarticulación de dichas bandas criminales, la cotidianidad de los haitianos se vio normalizada, en cierta medida y sus riesgos a la integridad física neutralizada. No obstante, los perjuicios para la seguridad de los haitianos se observan en el costo que tuvo, en vidas humanas civiles, esta lucha contra las bandas delincuenciales, lo que hace que los familiares de las víctimas no puedan tener un desarrollo completo, a causa de la 
pérdida de sus seres queridos y, además, sientan miedo hacia lo que pueda pasar con sus propias vidas.

La situación de salud pública que conllevó la epidemia de cólera es también una pérdida de la seguridad humana de los haitianos, puesto que el alto ratio de muertes que ha dejado la enfermedad, supone un riesgo alto para todas las partes de la seguridad de cada uno de los habitantes de Haití. A su vez, sumado al riesgo que hay para sus vidas a causa de la epidemia, está el riesgo al que deben verse enfrentados debido a la constante violación a los derechos humanos realizados por los soldados de la MINUSTAH. Dichos riesgos, generados principalmente por la misión de la $\mathrm{ONU}$, hace que la seguridad humana de los haitianos no se haya visto del todo protegida por la misión, pese a que esta era su tarea primordial, mediante el cumplimiento de sus tres objetivos básicos.

Sin embargo, la misión consiguió al menos que la situación en el país fuese lo suficientemente aceptable para la realización de las elecciones presidenciales que tuvieron lugar en el año 2006, lo que es una consecución de al menos uno de sus objetivos primordiales y que, a su vez, da una pequeña esperanza a los haitianos de que la situación dentro del país puede mejorar (como otra arista significativa del concepto de seguridad humana). No obstante, el trabajo de la MINUSTAH se hizo aun mayor (y sus aportes mucho más significativos), luego del terremoto que tuvo lugar en el año 2010.

Esto socavó los pequeños logros que había alcanzado la misión y supuso un detrimento exponencial en la seguridad humana de los haitianos, de forma que la misión tuvo que reformarse y expandir sus formas de trabajo para volver a generar una estabilización en el país (cosa que se ve actualmente reflejada con el establecimiento de la Misión de apoyo a la justicia en Haití -MINUJUSTH-). Siendo así, queda abierta a nuevas investigaciones la gestión que tuvo la MINUSTAH en el periodo inmediatamente posterior al terremoto, 
a fin de verificar si se han continuado sus patrones de acción, y si ha conseguido estabilizar o al menos mitigar, la crisis en Haití luego del desastre.

\section{Referencias Bibliográficas}

Banco Mundial. (2012). Homicidios intencionales (por cada 100.000 habitantes). Recuperado de: https://datos.bancomundial.org/indicador/VC.IHR.PSRC. P5?locations $=\mathrm{HT}$

Banco Mundial. (2016). Crecimiento de la población (porcentaje anual). Recuperado de: https://datos.bancomundial.org/indicador/SP.POP.GROW?locations=HT

Couceiro, E. (2005). Haití: la MINUSTAH, un mandato fuerte, una interpretación frustrante. Papeles, 92, 41-48. Recuperado de: http://www.cries.org/newsletter pdc/Haiti_MINUSTAH Couceiro.pdf

Dural, J. (2013). Las operaciones de paz de naciones unidas del capítulo VII: ¿Excepción o práctica extendida? Barcelona, España: Universitat pompeu fabra. Recuperado de: Dialnet.

Feltmann, A. \& Montes, J. (2008). Haití: tribulaciones de un Estado colapsado. Ciencia Política, 28(1), 245-264. Recuperado de: EBSCOhost

Fuentes, C. (2012). Seguridad humana: referencias conceptuales y enfoque práctico para América Latina. En: F. Rojas (Ed.), Seguridad Humana: nuevos enfoques (pp. 33-54). San José, Costa Rica: FLACSO. Recuperado de: http://www20.iadb.org/intal/catalogo/PE/2012/12042.pdf

González, M. \& Manero, A. (2011). El conflicto de Haití. Madrid, España. Universidad Carlos III de Madrid, Instituto de Estudios Internacionales y Europeos Francisco de Vitoria. Recuperado de: https://e-archivo.uc3m.es/bitstream/handle/10016/17390/14_ conflictos_haiti_2011.pdf

Grandin, G. \& Bhatt, K. (2011, septiembre, 26). 10 reasons why the UN occupation in Haiti must end. The Nation. Recuperado de: https://www.thenation.com/article/10reasons-why-un-occupation-haiti-must-end/ 
Le Chevalier, G. (2011). The "MINUSTAH experience". En: Heine, J. \& Thompson, A. (Ed.), Fixing Haiti: MINUSTAH and beyond (pp. 117-125). Nueva York, Estados Unidos de América: United Nations University Press. Recuperado de: EBSCOhost.

Mason, S. \& Rychard, S. (2005). Conflict analysis tools. Bern: Swiss Agency for Development and Cooperation.

Paris, R. (2010). Saving liberal peacebuilding. Review of International Studies, 36(2), 337365.

Roberts, D. (2012). Saving liberal peacebuilding from itself. Peace Review, 24(3), 366-373. Recuperado de: EBSCOhost

Serrano, E. (2006). Las operaciones para el mantenimiento de la paz en Haití. (1991-2005). Desafios, 16, 180-215. Recuperado de: Dialnet.

Torchiaro, L. (2007). MINUSTAH: una decisión estratégica con implicancias regionales. Bogotá, Colombia: Friedich Ebert Stiftung. Recuperado de: http://www.fes-seguridadregional.org/images/stories/docs/4110-001_g.pdf

Valdés, J. (2008). La MINUSTAH y la reconstrucción del Estado haitiano. Estudios Internacionales, 159, 129-142. Recuperado de: http://www.revistaei.uchile.cl/index. php/REl/article/viewFile/13924/14215

Wargny, G. (2005). ¿Ahora o nunca? Informe sobre la situación y los retos del país. BarceIona, España: Escola de Cultura de Pau. Recuperado de: http://escolapau.uab.cat/ img/programas/alerta/haiti/informe001.pdf

Zapata, J. (2014). La teoría del Estado fallido: entre aproximaciones y disensos. Relaciones Internacionales, Estrategia y Seguridad, 9(1), 87-110. Recuperado de: http://www. redalyc.org/pdf/927/92731211004.pdf 